\title{
Identification of novel non-coding RNAs in Rhizoctonia solani through mining of transcriptomic data
}

\author{
Balachander Durairaj ${ }^{1}$, Sudheesh K. Prabhudas ${ }^{2}$, Jaiganesh Rengarajan ${ }^{1}$, Iyappan Sellamuthu ${ }^{1 *}$ \\ ${ }^{1}$ Department of Genetic Engineering, Faculty of Engineering and Technology, SRM Institute of Science and Technology, Chennai, India. \\ ${ }^{2}$ Nutrition Genetics and Biotechnology Division NGBD ICAR, Central Institute of Brackishwater Aquaculture, Chennai, India.
}

\begin{tabular}{l}
\hline ARTICLE INFO \\
\hline Article history: \\
Received on: January 19, 2021 \\
Accepted on: June 27,2021 \\
Available online: September 20, 2021 \\
\hline
\end{tabular}

Key words:

Rhizoctonia solani, non coding RNA, lncRNA, transcriptome mining, plant microbe interaction

\begin{abstract}
Rhizoctonia solani is a plant pathogenic fungus infecting a wide range of hosts including economically important crops such as wheat, rice, and vegetables, etc. and leads to a loss in agricultural production. Various chemicals and bio fungicides are used to control $R$. solani. Understanding of gene expression and its function during host interaction will be useful to identify potential targets in R.solani for its effective control. In recent studies, non-coding RNAs (ncRNAs) are found to have a role in regulating cellular functions. In the current study, we report 16 ncRNAs from $R$. solani identified using raw transcriptomic data from three different bio projects reported in NCBI's sequence read archive database. The ncRNAs from F001 to ncRNA F0011 was expressed with fragments per kilo million reads (FPKM) values ranging from 100 to 20,000. Out of these 11 ncRNAs, 7 ncRNAs has the same intron splicing sites in all three bio projects. The ncRNA F0012 to ncRNA F0016 was found to be expressed approximately 10-80 FPKM and are present in all three bio projects, out of these five ncRNAs, three are found to have similar splicing sites in all three bio projects. The high expression levels of the ncRNAs and their presence in the genome confirmed by different datasets point to the fact that they might have a major function in the organism and should be studied further to characterize it functionally and the current study might serve as the first step to achieve it.
\end{abstract}

\section{INTRODUCTION}

Rhizoctonia solani is a plant pathogenic fungus which belongs to phylum Basidiomycota, has a wide range of host and causes major loss in food crops. Rhizoctonia solani is divided into 14 anastomosis groups based on their anastomosis behavior (AG1AG13 and AGBI) [1]. AG1 is again classified into six subgroups (IA, IB, IC, ID, IE, and IF) [2-4], Rice sheath blight is majorly caused by intraspecific group IA and studied a lot due to the economic importance of the host [5-7]. Rhizoctonia solani AG1-IA genome from NCBI (Accession No: GCA_000334115.1) is 36.94 $\mathrm{Mb}$ long and has 10489 protein sequences, and is widely used in transcriptome analysis for $R$. solani AG1-IA [8]. RNASeq studybased evidence suggests that some of the regions in this genome are highly expressed but are not annotated. This partial annotation

*Corresponding Author

Iyappan Sellamuthu, Department of Genetic Engineering, Faculty of Engineering and Technology, SRM Institute of Science and Technology, Chennai, India.E-mail: iyappansbt@gmail.com might lead to the omission of certain important gene expression during the study of host infection. In the current study, the existing transcriptome data for $R$. solani for their host interaction were analyzed and found that certain non-coding RNAs (ncRNAs) gets expressed during pathogenesis fragments per kilo million reads (FPKM).

NcRNAs that do not code any proteins are widely studied recently [9]. The role of ncRNA in plants and animal were shown to be involved in their immunity during infection [10]. Some mobile ncRNA were reported in the fungal pathogen and their mechanism through entering into the host cell and suppress the genes related to immunity [11]. These ncRNAs are of two types, long noncoding RNAs (lncRNAs) which are above 200 nucleotides long, and small non-coding (sncRNAs) with less than 200 nucleotides long. These lncRNAs are transcribed by DNA polymerase 2 from the genome, similar to mRNA and also they undergo $5^{\prime}$ capping, splicing, and poly-A tailing [12]. Most of the IncRNAs transcribed from ORF had only a single peptide match, but very few lncRNAs contains cryptic ORF [13]. IncRNAs in yeast is found to have 
a functional role like regulating gene expression, response to environment and chromosome pairing, etc $[14,15]$. Swr1, Isw2, Rsc, and Ino80 are distinct chromatin remodeling complexes in Saccharomyces cerevisiae that are global repressors of lncRNA transcription [16].

Here, SRR files from NCBI were downloaded and the differentially expressed genes were analyzed to discover certain unclassified genes in the $R$. solani AG1-IA genome to be expressed significantly that has an important biological and molecular function during infection. Also, differentially expressed lncRNAs and sncRNAs were identified and studied. This finding can help in improving the study of lncRNA, function, and metabolism in plant photogenic fungi.

\section{METHODS}

\subsection{Sequence Read Archive (SRA) Files Selected for the Study}

The NCBI SRA tool kit was used to download the RNA Seq raw data from SRA database and under bio project numbers PRJNA298635, PRJNA369092, and PRJNA377841. A total of 11 conditions were selected from these three bio projects, $1 \mathrm{C} 1(R$. solani AG1-IA infected on Tetep, 1DPI), 1_C2 ( $R$. solani AG1-IA infected on TP309, 1DPI), 1_C3 ( $R$. solani AG1-IA infected on PB1, 1DPI), 1_C4 (R. solani AG1-IA infected on Tetep, 3DPI), 1 C5 ( $R$. solani AG1-IA infected on TP309, 3DPI), 1 C6 $(R$. solani AG1-IA infected on PB1, 3DPI), 2 C1 (R. solani AG1-IA infected on soya leaf, the onset of necrosis), 2 C2 (R. solani AG1IA infected on soya leaf, post-onset of necrosis), 3 C1 ( $R$. solani AG1-IA infected on soya leaf), 3_C2 ( $R$. solani AG1-IA infected on maize leaves) and $3 \mathrm{C} 3(R$. solani AG1-IA infected on rice leaf) [17-19].

\subsection{Mining of Transcriptomic Data for Non-Coding RNA}

The raw reads were analyzed for quality and adapter contamination using FastQC (version-0.11.9) [20]. The low-quality reads and adapters were trimmed using trimmomatic (version-0.39) [21] and reads with length below 50 nucleotides were dropped. The filtered reads were then mapped to $R$. solani AG1-IA genome (GCA 000334115.1) using tophat (version-2.1.1) [22]. The mapped reads were then processed with cufflinks (version-2.2.1), cufflinks (version-1.0.0) and cuffdiff (version-2.2.1) [23]. These genes were traced back to the genome and the sequence was retrieved using bed tools (version-2.27.1) [24]. The obtained sequences were locally blasted against the NR database using blastx (version-2.9.0+) [25].

\subsection{Identification of ncRNAs}

The unannotated genes were further filtered to remove transcriptional noise by avoiding single-exon genes and genes with FPKM value $<0.5$. The differently expressed genes with $p$ value less than 0.5 were taken for identification of long non-coding. The sequences above 200 nucleotides were selected for identifying lncRNAs. The coding potential was calculated using CPC2, CNIT, and PLEK tools [26-28]. The sequences were considered as noncoding if all the three tools predicted it as non-coding [29]. These genes were further filtered by the presence of one gene in more than eight conditions or FPKM above 100, which is present in at least four conditions and more than one bio project transcriptomic data.

\section{RESULTS AND DISCUSSION}

\subsection{Mining of Transcriptomic Data for Non-Coding RNA}

LncRNA have vital role in regulation of gene expression and associated with many diseases such as cancer [30]. It also plays a crucial role in developing immunity against various pathogen in plants and animals [31]. In the present study, a total of 26 transcriptome data for $R$. solani interaction with host were downloaded from all three bio projects and the quality trimed reads were mapped to the genome. The raw read counts after trimming and the percentage of reads mapped to the genome are listed in Table 1. The percentage of the reads mapped to the genome varies drastically between the SRA files as per Table 1 . The reason could be that the SRA data is mostly mixed with the host transcriptomic data, which accounts for the major portion of these files. The RNA Seq analysis using tophat, cufflinks, and cuffdiff revealed 2,448 unclassified genes in the reference genome and the sequences were retrieved from the genome using bed tools. Among the noncoding transcripts, the one with more than $200 \mathrm{nt}$ are classified as long ncRNAs and are considered for further analysis [32]. The annotation of these sequences using blastx against nr database annotated 1,868 sequences, and the remaining 580 sequences had no blast hits. These 580 sequences either have no predecessor sequences in the NR database or they might be potential noncoding sequences.

\subsection{Identification of ncRNAs}

The 580 genes with no blast hits were further filtered and analyzed for their coding potential using CPC2, CNIT, and PLEK. The tools predicted 536, 556, and 569 non-coding sequences with CPC2, CNIT, and PLEK, respectively. The 511 sequences predicted as non-coding by all the three tools as depicted in Figure 1 were considered for further analysis. The predicted potential non-coding sequences were further screened based on their FPKM values and the number of conditions they were present in and narrowed it down to 16 sequences. These 16 sequences were finalized as ncRNA. Out of this, 5-ncRNA was differently expressed in $\geq 8$ conditions with FPKM less than 100, 11ncRNA was found to be expressed with FPKM values more than 100 ranging up to 20,000 and are present in more than one bio project transcriptomic data. Ten ncRNA retains similar splice sites in all three bioprojects and presented in Table 4. These results were supported by Nitsche et al. [33] who reported that there is a conserved splice site among the ncRNAs and lead to track the evolutionary changes. The details of the number of conditions the ncRNAs were expressed, the number of bio projects, and the type of ncRNA for the ncRNAs having FPKM values above 100 and below 100 are given in Tables 2 and 3, respectively. There are numerous reports stating lncRNAs have a role in regulatory mechanism in gene expression level and the same were reviewed recently [34]. Wang et al. [35] reported that 161 long non coding RNAs were differentially expressed in rice responsive to Blast fungal infection. This also supports the 
Table 1: List of SRA files included in the study.

\begin{tabular}{|c|c|c|c|c|c|}
\hline S. no. & Bio project & SRR accession No. & $\begin{array}{c}\text { Total reads before } \\
\text { trimming }\end{array}$ & $\begin{array}{l}\text { Total reads after } \\
\text { trimming }\end{array}$ & $\begin{array}{l}\text { Reads mapped to genome } \\
(\%)\end{array}$ \\
\hline 1 & Bio project 1 & SRR2854160 & $28,511,520$ & $28,511,520$ & $61.1 \%$ \\
\hline 2 & & SRR2859034 & $31,832,558$ & $31,832,558$ & $37.9 \%$ \\
\hline 3 & & SRR2859785 & $35,111,684$ & $35,111,684$ & $58.8 \%$ \\
\hline 4 & & SRR2873752 & $28,967,576$ & $28,967,576$ & $12.6 \%$ \\
\hline 5 & & SRR2906372 & $38,624,268$ & $38,624,268$ & $17.4 \%$ \\
\hline 6 & & SRR2906726 & $40,052,168$ & $40,052,168$ & $2.5 \%$ \\
\hline 7 & & SRR9044112 & $38,404,774$ & $38,404,774$ & $29.0 \%$ \\
\hline 8 & & SRR9044119 & $43,550,864$ & $43,550,864$ & $5.4 \%$ \\
\hline 9 & Bio project 2 & SRR5209772 & $35,087,068$ & $31,203,980$ & $76.0 \%$ \\
\hline 10 & & SRR5209773 & $31,206,092$ & $26,117,842$ & $75.4 \%$ \\
\hline 11 & & SRR5209774 & $28,346,372$ & $25,616,466$ & $76.3 \%$ \\
\hline 12 & & SRR5209775 & $22,617,670$ & $20,136,584$ & $73.9 \%$ \\
\hline 13 & & SRR5209776 & $29,720,586$ & $27,091,046$ & $73.9 \%$ \\
\hline 14 & & SRR5209777 & $26,818,988$ & $23,161,090$ & $73.6 \%$ \\
\hline 15 & & SRR5209778 & $24,634,370$ & $22,206,974$ & $69.5 \%$ \\
\hline 16 & & SRR5209779 & $28,176,672$ & $23,151,690$ & $68.1 \%$ \\
\hline 17 & & SRR5209780 & $27,320,880$ & $24,483,114$ & $70.2 \%$ \\
\hline 18 & & SRR5209781 & $27,030,046$ & $24,526,572$ & $70.7 \%$ \\
\hline 19 & & SRR5209782 & $30,295,896$ & $27,285,188$ & $70.9 \%$ \\
\hline 20 & & SRR5209783 & $24,009,354$ & $22,436,774$ & $71.3 \%$ \\
\hline 21 & Bio project 3 & SRR5500529 & $26,587,034$ & $25,944,658$ & $68.1 \%$ \\
\hline 22 & & SRR5500530 & $28,289,406$ & $27,655,028$ & $67.9 \%$ \\
\hline 23 & & SRR5500532 & $26,958,568$ & $26,296,358$ & $73.1 \%$ \\
\hline 24 & & SRR5500533 & $24,732,110$ & $24,217,014$ & $73.4 \%$ \\
\hline 25 & & SRR5500534 & $23,405,190$ & $22,787,166$ & $78.0 \%$ \\
\hline 26 & & SRR5500535 & $29,428,220$ & $28,834,604$ & $78.2 \%$ \\
\hline
\end{tabular}

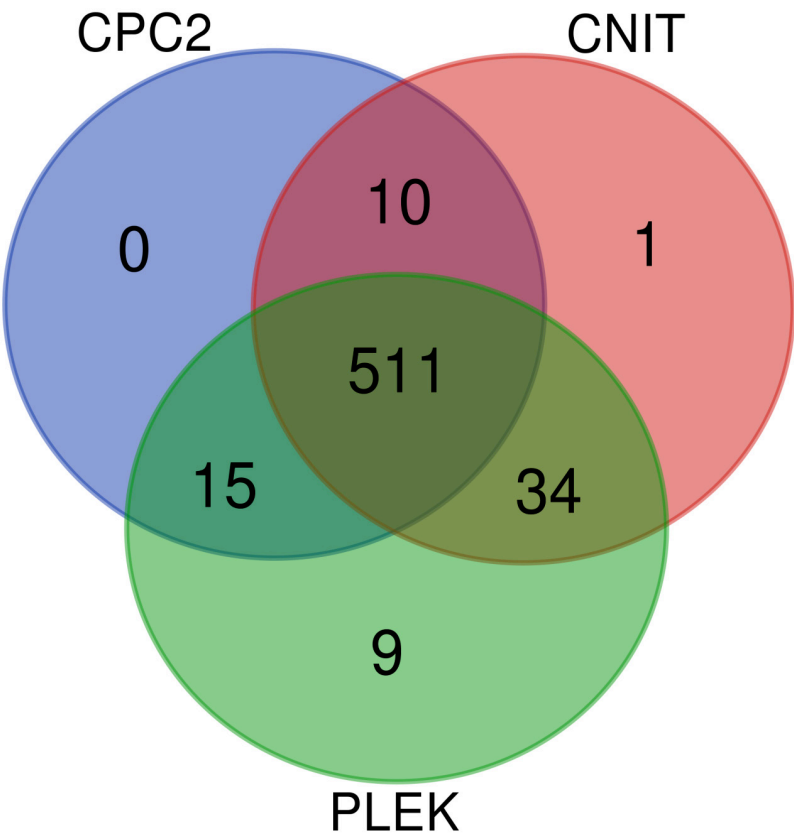

Figure 1: Coding potential calculation using CPC2, CNIT, and PLEK. 
Table 2: List of predicted ncRNA expressed with FPKM more than 100.

\begin{tabular}{ccccc} 
NC-RNA & Locus & $\begin{array}{c}\text { Number of conditions } \\
\text { has expression }\end{array}$ & $\begin{array}{c}\text { Bioprojects containing } \\
\text { the expression }\end{array}$ & $\begin{array}{c}\text { NC-RNA } \\
\text { type }\end{array}$ \\
NC-RNA F001 & KB317696.1:824260-827451 & 8 & 3 & LNC-RNA \\
NC-RNA F002 & KB317698.1:97800-98802 & 8 & 3 & LNC-RNA \\
NC-RNA F003 & KB317700.1:980840-981146 & 7 & 2 & SNC-RNA \\
NC-RNA F004 & KB317701.1:439656-439959 & 3 & 2 & SNC-RNA \\
NC-RNA F005 & KB317701.1:483929-485975 & 8 & 3 & LNC-RNA \\
NC-RNA F006 & KB317705.1:471573-471886 & 10 & 3 & LNC-RNA \\
NC-RNA F007 & KB317706.1:637144-637947 & 8 & 3 & LNC-RNA \\
NC-RNA F008 & KB317710.1:116289-116584 & 8 & 3 & LNC-RNA \\
NC-RNA F009 & KB317726.1:101314-107653 & 7 & 3 & LNC-RNA \\
NC-RNA F0010 & KB317748.1:130750-131698 & 10 & 3 & LNC-RNA \\
NC-RNA F0011 & KB320145.1:0-237 & 4 & 2 & SNC-RNA \\
\hline
\end{tabular}

Table 3: List of predicted ncRNA that are expressed in more than or equal to eight conditions with FPKM less than 100.

\begin{tabular}{ccccc} 
NC-RNA & Locus & $\begin{array}{c}\text { Number of conditions } \\
\text { has expression }\end{array}$ & $\begin{array}{c}\text { Bioprojects containing } \\
\text { the expression }\end{array}$ & $\begin{array}{c}\text { NC-RNA } \\
\text { type }\end{array}$ \\
NC-RNA F0012 & KB317707.1:280114-281077 & 10 & 3 & LNC-RNA \\
NC-RNA F0013 & KB317709.1:358322-358980 & 8 & 2 & LNC-RNA \\
NC-RNA F0014 & KB317711.1:412940-413725 & 9 & 3 & LNC-RNA \\
NC-RNA F0015 & KB317725.1:115863-118971 & 8 & 3 & LNC-RNA \\
NC-RNA F0016 & KB317744.1:164906-167799 & 9 & 3 & LNC-RNA \\
\hline
\end{tabular}

Five ncRNA was identified, out of that one was found to be expressed in ten conditions from three bio projects, two ncRNA was expressed in nine conditions from three bio projects and two was expressed in eight conditions.

Table 4: The splicing sites for 10 ncRNAs are exactly same in all three bio projects that is the intron region is common for these ncRNAs that are listed with the exon regions.

\begin{tabular}{|c|c|c|c|c|c|}
\hline \multirow[t]{2}{*}{ SI. no. } & \multirow[t]{2}{*}{ NC-RNA } & \multirow[t]{2}{*}{ Accession } & \multicolumn{2}{|c|}{ Exon } & \multirow[t]{2}{*}{ NC-RNA type } \\
\hline & & & Start & End & \\
\hline \multirow[t]{2}{*}{1} & NC-RNA F001 & KB317696.1 & 824,261 & 824,407 & LNC-RNA \\
\hline & & & 824,458 & 824,644 & \\
\hline \multirow[t]{3}{*}{2} & NC-RNA F002 & KB317698.1 & 97,811 & 98,013 & LNC-RNA \\
\hline & & & 98,072 & 98,163 & \\
\hline & & & 98,304 & 98,487 & \\
\hline \multirow[t]{2}{*}{3} & NC-RNA F005 & KB317701.1 & 483,930 & 484,058 & LNC-RNA \\
\hline & & & 484,110 & 484,342 & \\
\hline \multirow[t]{2}{*}{4} & NC-RNA F006 & KB317705.1 & 471,574 & 471,729 & LNC-RNA \\
\hline & & & 471,781 & 471,887 & \\
\hline \multirow[t]{2}{*}{5} & NC-RNA F008 & KB317710.1 & 116,290 & 116,376 & LNC-RNA \\
\hline & & & 116,436 & 116,585 & \\
\hline \multirow[t]{2}{*}{6} & NC-RNA F009 & KB317726.1 & 107,184 & 107,387 & LNC-RNA \\
\hline & & & 107,444 & 107,654 & \\
\hline \multirow[t]{2}{*}{7} & NC-RNA F0011 & KB320145.1 & 1 & 42 & SNC-RNA \\
\hline & & & 111 & 218 & \\
\hline \multirow[t]{3}{*}{8} & NC-RNA F0013 & KB317709.1 & 358,323 & 358,556 & LNC-RNA \\
\hline & & & 358,621 & 358,707 & \\
\hline & & & 358,762 & 358,847 & \\
\hline \multirow[t]{2}{*}{9} & NC-RNA F0014 & KB317711.1 & 412,941 & 413,436 & LNC-RNA \\
\hline & & & 413,488 & 413,560 & \\
\hline \multirow[t]{2}{*}{10} & NC-RNA F0015 & KB317725.1 & 115,864 & 115,916 & LNC-RNA \\
\hline & & & 116,105 & 116268 & \\
\hline
\end{tabular}


regulatory functions of identified lncRNAs in $R$. solani during its interaction with host plants.

\section{CONCLUSION}

The SRA database of NCBI contains an immense amount of raw data with untapped potential, much of the data has been generated by taking a single aspect of an experimental setup. We have successfully utilized some of the RNASeq based raw data available for identifying certain Novel lncRNA's that are significantly expressed by $R$. solani. We have identified 11 ncRNAs with FPKM values ranging from 100 to 2,000 and 5 ncRNAs with FPKM values between 10 and 80. However the functional characterization has not been conducted for these lncRNAs, their prediction using the raw data might trigger the interest of the scientific community to utilize the available data to identify some of the previously unannotated genes or ncRNAs. Some of these ncRNAs have very high expression rate and their prediction based on data generated from different groups itself serves as a validation of their presence in the genome. The role and mechanism of the non-coding RNA were reviewed recently and explained its role in plant immunity and pathogenesis [36]. Extending the studies on the function and mechanism of these lncRNA will helps to understand more mechanism underlying the pathogenesis and will create a novel methods for its control.

\section{ACKNOWLEDGMENTS}

The authors would like to thank SRMIST for providing opportunity to carry out this research work in the SRM-HPC facility.

\section{AUTHOR CONTRIBUTIONS}

All authors made substantial contributions to conception and design, acquisition of data, or analysis and interpretation of data; took part in drafting the article or revising it critically for important intellectual content; agreed to submit to the current journal; gave final approval of the version to be published; and agree to be accountable for all aspects of the work. All the authors are eligible to be an author as per the international committee of medical journal editors (ICMJE) requirements/guidelines.

\section{FUNDING}

There is no funding to report.

\section{CONFLICTS OF INTEREST}

The authors report no financial or any other conflicts of interest in this work.

\section{ETHICAL APPROVALS}

This study does not involve experiments on animals or human subjects.

\section{REFERENCES}

1. Ogoshi A. Ecology and pathogenicity of anastomosis and intraspecific groups of Rhizoctonia solani Kuhn. Annu Rev Phytopathol. 1987;25(1):125-43.
2. Farrokhi-Nejad R, Cromey MG, Moosawi-Jorf SA. Determination of the anastomosis grouping and virulence of Rhizoctonia spp. associated with potato tubers grown in Lincoln, New Zealand. Pak J Biol Sci 2007;10(21):3786-93.

3. Priyatmojo A, Escopalao VE, Tangonan NG, Pascual CB, Suga H, Kageyama K, et al. Characterization of a new subgroup of Rhizoctonia solani anastomosis group 1 (AG-1-ID), causal agent of a necrotic leaf spot on coffee. Phytopathology 2001;91(11):1054-61.

4. Godoy-Lutz G, Kuninaga S, Steadman JR, Powers K. Phylogenetic analysis of Rhizoctonia solani subgroups associated with web blight symptoms on common bean based on ITS-5.8S rDNA. J Gen Plant Pathol 2008;74(1):32-40.

5. Khodayari M, Safaie N, Shamsbakhsh M. Genetic diversity of Iranian AG1-IA isolates of Rhizoctonia solani, the cause of rice sheath blight, using morphological and molecular markers. J Phytopathol 2009;157(11-12):708-14.

6. Tan W, Zhang W, Ou Z, Li C, Zhou G, Wang Z, et al. Analyses of the temporal development and yield losses due to sheath blight of rice (Rhizoctonia solani AG1.1a). Agric Sci China 2007;6(9):1074-81.

7. Nadarajah K, Razali NM, Cheah BH, Sahruna NS, Tathode M, Bankar $\mathrm{K}$. Draft genome sequence of Rhizoctonia solani anastomosis group 1 subgroup 1A strain 1802/KB isolated from rice. Genome Announc 2009;5(12):1-2.

8. Zheng A, Lin R, Zhang D, Qin P, Xu L, Ai P, et al. The evolution and pathogenic mechanisms of the rice sheath blight pathogen. Nat Commun 2013;4:1410-24.

9. Okazaki Y, Furuno M, Kasukawa T, Adachi J, Bono H, Kondo S, et al. Analysis of the mouse transcriptome based on functional annotation of 60,770 full-length cDNAs. Nature 2002;420(6915):563-73. Available via http://www.informatics.jax.org/mgihome/ (Accessed 11 September 2020)

10. Dhingra S. Role of non-coding RNAs in fungal pathogenesis and antifungal drug responses. Curr Clin Microbiol Rep 2020;7:133-41.

11. Huang CY, Wang H, Hu P, Hamby R, Jin H. Small RNAs - big players in plant-microbe interactions. Cell Host Microbe 2019;26(2):173-182.

12. Guttman M, Amit I, Garber M, French C, Lin MF, Feldser D, et al. Chromatin signature reveals over a thousand highly conserved large non-coding RNAs in mammals. Nature 2009;458(7235):223-7. Available via https://www.nature.com/articles/nature07672 (Accessed 11 September 2020)

13. Bánfai B, Jia $\mathrm{H}$, Khatun J, Wood E, Risk B, Gundling WE, et al. Long noncoding RNAs are rarely translated in two human cell lines. Genome Res 2012;22(9):1646-57. Available via http://pmc/articles/ PMC3431482/?report=abstract (Accessed 11 September 2020)

14. Yamashita A, Shichino Y, Yamamoto M. The long non-coding RNA world in yeasts. Biochim Biophys Acta Gene Regul Mech 2016;1859:147-54.

15. Houseley J, Rubbi L, Grunstein M, Tollervey D, Vogelauer M. A ncRNA modulates histone modification and mRNA induction in the yeast GAL gene cluster. Mol Cell. 2008;32(5):685-95.

16. Alcid EA, Tsukiyama T. ATP-dependent chromatin remodeling shapes the long noncoding RNA landscape. Genes Dev 2014;28(21):2348 60. Available via http://pmc/articles/PMC4215180/?report=abstract (Accessed 11 September 2020)

17. Ghosh S, Kanwar P, Jha G. Identification of candidate pathogenicity determinants of Rhizoctonia solani AG1-IA, which causes sheath blight disease in rice. Curr Genet 2018;64(3):729-40; doi:10.1007/ s00294-017-0791-7

18. Copley TR, Duggavathi R, Jabaji S. The transcriptional landscape of Rhizoctonia solani AG1-IA during infection of soybean as defined by RNA-seq. PLoS One 2017;12(9):1-22.

19. Xia Y, Fei B, He J, Zhou M, Zhang D, Pan L, et al. Transcriptome analysis reveals the host selection fitness mechanisms of the Rhizoctonia solani AG1IA pathogen. Sci Rep 2017;7(1):1-16; doi:10.1038/s41598-017-10804-1 
20. Andrews S. FASTQC. A quality control tool for high throughput sequence data.

21. Bolger AM, Lohse M, Usadel B. Trimmomatic: a flexible trimmer for Illumina sequence data. Bioinformatics. 2014;30(15):2114-20.

22. Trapnell C, Pachter L, Salzberg SL. TopHat: discovering splice junctions with RNA-Seq. Bioinformatics. 2009;25(9):1105-11.

23. Trapnell C, Roberts A, Goff L, Pertea G, Kim D, Kelley DR, et al. Differential gene and transcript expression analysis of RNA-seq experiments with TopHat and Cufflinks. Nat Protoc 2012;7(3): 562-78.

24. Quinlan AR, Hall IM. BEDTools: a flexible suite of utilities for comparing genomic features. Bioinformatics 2010;26(6):841-2.

25. Camacho C, Coulouris G, Avagyan V, Ma N, Papadopoulos J, Bealer $\mathrm{K}$, et al. BLAST+: architecture and applications. BMC Bioinformatics 2009; 10:1-9.

26. Kang YJ, Yang DC, Kong L, Hou M, Meng YQ, Wei L, et al. CPC2: a fast and accurate coding potential calculator based on sequence intrinsic features. Nucleic Acids Res 2017;45(W1):W12-6.

27. Guo JC, Fang SS, Wu Y, Zhang JH, Chen Y, Liu J, et al. CNIT: a fast and accurate web tool for identifying protein-coding and long noncoding transcripts based on intrinsic sequence composition. Nucleic Acids Res 2019;47(W1):W516-22.

28. Li A, Zhang J, Zhou Z. PLEK: a tool for predicting long non-coding RNAs and messenger RNAs based on an improved k-mer scheme. BMC Bioinformatics 2014;15(1):311.

29. Li S, Yu X, Lei N, Cheng Z, Zhao P, He Y, et al. Genome-wide identification and functional prediction of cold and/or droughtresponsive lncRNAs in cassava. Sci Rep 2017;7(March):1-15.
30. Carlevaro-Fita J, Lanzós A, Feuerbach L. Cancer LncRNA census reveals evidence for deep functional conservation of long noncoding RNAs in tumorigenesis. Commun Biol 2020;3:56.

31. Feng Q, Li Y, Zhao ZX, Wang WM. Contribution of small RNA pathway to interactions of rice with pathogens and insect pests. Rice 2021;14(1):1-5.

32. Sacco LD, Baldassarre A, Masotti A. Bioinformatics tools and novel challenges in long non-coding RNAs (lncRNAs) functional analysis. Int J Mol Sci 2012;13(1):97-114.

33. Nitsche A, Rose D, Fasold M, Reiche K, Stadler PF. Comparison of splice sites reveals that long noncoding RNAs are evolutionarily well conserved. RNA 2015;21(5):801-12.

34. Heo JB, Lee YS. Molecular functions of long noncoding transcripts in plants. J Plant Biol 2015;58:361-5.

35. Wang LL, Jin JJ, Li LH, Qu SH. Long non-coding RNAs responsive to blast fungus infection in rice. Rice 2020;13:77.

36. SongL, Fang Y, Chen L, Wang J, Chen X. Role of non-coding RNAs in plant immunity. Plant Commun 2021;2(3):100180.

\section{How to cite this article:}

Durairaj B, Prabhudas SK, Rengarajan J, Sellamuthu I. Identification of novel non-coding RNAs in Rhizoctonia solani through mining of transcriptomic data. J Appl Biol Biotech 2021;9(S1):7-12. 